\title{
Special Certification for General Surgical Oncology: Concerns of a Curmudgeon
}

\author{
Walter Lawrence Jr., MD \\ Division of Surgical Oncology and Massey Cancer Center, Virginia Commonwealth University, Richmond, VA
}

The possibility of some form of official recognition for the general surgeon who has expertise in surgical oncology has been discussed ever since the evolution of the Society of Surgical Oncology (SSO) from the James Ewing Society back in 1975. The Council of the SSO concluded in the late 1970s that this recognition should definitely take the form of certificates for those who had additional competence and experience in the surgical and overall management of patients with cancer for whom general surgery operations played some role. After all, our fellow disciplines of medical oncology and radiation oncology had established such a process and it seemed logical for surgeons participating in multidisciplinary care of cancer patients to have similar recognition as experts in oncology. Since I had the privilege of serving as SSO President then, I was instructed to relay this message from our SSO Council to my colleagues on the American Board of Surgery (ABS). Due to a general reticence for such subspecialization at that time among members of the ABS, and possibly for other reasons, this proposal from the SSO fell on "deaf ears" and nothing happened.

Three decades later, much has changed in terms of the maturation of the field of surgical oncology within general surgery. Also, the attitudes of our general surgical leaders regarding the suitability of formal certification of general surgeons with special competence in surgical oncology and other specialized areas has changed. The ABS now seems poised to endorse official recognition for general surgeons who have had specialized training in surgical oncology. They propose this by establishing special competence through training guidelines and a formal examination process. Since the leadership and the majority of the SSO

(C) Society of Surgical Oncology 2011

Published Online: 22 April 2011

W. Lawrence Jr., MD

e-mail: WLawrence@MCVH-VCU.EDU have been urging these developments over several decades, there is considerable jubilation about these recent developments despite a few negative views expressed by some of us. Some potential adverse effects of formal certification are not completely dismissed by those favoring this process, but the statement often made at present is that the positives outweigh the negatives. My purpose for writing this opinion piece is to suggest the opposite-that the negatives of this development may overwhelm possible advantages achieved by certification of general surgical oncologists.

Regrettably, we health care providers are becoming more and more an industry and less and less an altruistic profession. For this reason, we are beginning to discuss "professionalism" in our education and training programs much more often, but the focus of medical practice has been "market share" and other business practices despite our lofty discussions of the importance of team efforts in patient care. It is for this reason that I strongly believe any certificate of special competence in a clinical health care environment can, and possibly will, be used by some hospitals through their bylaws to restrict the performance of patient care procedures to the "chosen few" with special competencies. It is clear that this is a good thing in many instances, particularly where craniotomy or prostatectomy is required, or even a technically demanding vascular operation. However, the overwhelming number of disorders that are appropriately cared for by surgical oncologists are procedures for which we routinely provide training and establish competence for surgeons completing approved general surgical training programs. Most of us agree that the well-trained general surgeon can deal with the overwhelming majority of these and the benefits provided to society by surgical oncologists are more in the leadership and cognitive areas, and much less in the technical sphere.

We do need surgical oncologists' leadership in clinical trials and for the overall management of very challenging 
and unusual oncologic problems. Despite this, we do not want the general surgeons who we have adequately trained to manage most general surgical cancer problems to be prevented in the future from performing this care because a "certified surgical oncologist" has taken over in his or her community hospital. I believe that the bottom line is that this formal certification process will unfairly restrict "fair trade" when it comes to surgical care in many communities. Not only does this seem unfair to the well-trained general surgeon practicing in our communities, but a waste of a valuable resource that we are currently producing: the well-trained, board-certified general surgeon. Despite some positive features to certification in surgical oncology, I believe this potential future restriction of privileges for surgical cancer care negates any advantage achieved by such a process. Also, I sincerely believe that we can continue to achieve adequate processes for establishing standards for the training and practice of surgical oncology within our Society of Surgical Oncology without creating an official certificate for the office wall. 